\title{
Contrato fijo de obra: fijación y revelado de una especial figura contractual en la era digital
}

\section{Fixed contract for a specific work in the construction industry: fixing and developing the image of an special employment contract in the digital age}

\author{
David Lantarón Barquín* \\ Catedrático de Derecho del Trabajo y la Seguridad Social \\ Universidad Cantabria \\ ORCID ID: 0000-0002-7851-4066
}

Recibido: 20/4/2021

Aceptado: $12 / 5 / 2021$

doi: https://doi.org/10.20318/labos.2021.6216

Resumen: $\quad$ El contrato fijo de obra, estandarte del sector de la construcción, es una figura controvertida en su naturaleza, que representa como acaso pocas otras el choque entre la categorización jurídica y la realidad negocial y del mercado de trabajo, avalada ésta, en principio, por el fin de tutela del trabajador. Con antecedentes en la Ordenanza de la Construcción, Vidrio y Cerámica del año 70 y anclaje en la LSC y en el ET, entre otras normas, ha sido atendida en el nivel negocial estatal ininterrumpidamente, destacando su cuestionada condición de modulación del contrato de obra o servicio determinado, refrendada no obstante por nuestro Tribunal Supremo, y el singular régimen de duración del contrato. Un tratamiento jurídico que deja tras de sí un reguero de incertidumbres, que conjuntamente con los pronunciamientos judiciales, son abordadas en el presente estudio.

Palabras clave: contrato fijo de obra, contrato de obra o servicio determinado, construcción.

Abstract: $\quad$ The fixed contract for a specific work in the construction industry, a flagship of this sector, is a controversial figure in its nature, which represents, as perhaps few others, the clash between legal categorisation and the reality of the business and labour market, the latter backed, in principle, by the aim of protecting the worker. With antecedents in the Construction, Glass and Ceramics Ordinance of 1970 and anchored in the LSC and in the ET, among other regulations, it has been uninterruptedly dealt with at the state negotiating level, highlighting its questioned condition of modulation of the contract for a specific work or service, endorsed however by our Supreme Court, and the singular regime of duration of the contract. A legal treatment that leaves behind a trail of uncertainties, which, together with the pronouncements of the courts, are addressed in this study.

Keywords: $\quad$ fixed contract for a specific work, employment contract for a specific work or service, construction industry.

*lantarond@unican.es 


\section{Escenario jurídico del contrato fijo de obra}

El llamado contrato fijo de obra es una realidad presente en el panorama jurídico-laboral del sector de la construcción de nuestro país desde hace ya tiempo. El propio Tribunal Supremo advierte en su Sentencia (Social) de 30 junio 2005 (R. Ud. 2426/2004) ${ }^{1}$, señera en esta materia, que el régimen convencional asume "lo que constituye en dicho sector una antigua y pacifica práctica contractual, la prestación por el trabajador fijo de obra de sucesivos servicios en otras distintas de la contratada, que ya encontraba apoyo legal en el art. 43 de la antigua y derogada Ordenanza de Trabajo de la Construcción del año 70". Ordenanza del Trabajo en la Construcción, Vidrio y Cerámica, de 28 de agosto de 1970, que es, en especial sus artículos 42 y 43, relevante, pese a su derogación, para entender el régimen jurídico actual de esta figura ${ }^{2}$.

Contrato fijo de obra cuya trascendencia se ve acrecentada por la importancia histórica de este sector en España. Sector que "ha sido durante décadas el principal trampolín de la economía y el instrumento generador básico de ocupaciones en nuestro país", entrando en una acusada recesión a partir del año 2008, motivada por la crisis financiera. Una recesión que revierte ligeramente desde el ańo 2014 y más claramente en el año 2018, pero que encuentra un nuevo y trascendente paso atrás desde el RD 463/2020, de 14 de marzo, por el que se declara el estado de alarma para la gestión de la situación de crisis sanitaria ocasionada por el COVID-19 (BOE de idéntica fecha). Siendo igualmente de destacar el cambio de rumbo del sector hacia un modelo más sostenible iniciado en la Ley 2/2011, de 4 de marzo, de Economía Sostenible (BOE, 5 de marzo) ${ }^{3}$.

Se trata, pese a ello y sin lugar a la más mínima duda, de una realidad controvertida, si bien amparada por la legislación estatal en la DA tercera Ley 32/2006, de 18 de octubre, reguladora de la subcontratación en el sector de la construcción (BOE, 19-X), de rúbrica "Negociación colectiva y calidad en el empleo". Ley que vino "a sancionar legalmente la antigua práctica convencional" ${ }^{4}$. Una realidad controvertida por su cuestionado quebranto a la causalidad de la contratación temporal y por la influencia que tiene en la altísima tasa de temporalidad del sector.

Disposición Adicional que, "con el objetivo de mejorar la calidad en el empleo de los trabajadores que concurren en las obras de construcción y, con ello, mejorar su salud y seguridad laborales", habilita a la negociación colectiva de ámbito estatal del sector de la construcción para "adaptar la modalidad contractual del contrato de obra o servicio determinado prevista con carácter general mediante fórmulas que garanticen mayor estabilidad en el empleo de los trabajadores, en términos análogos a los actualmente regulados en dicho ámbito de negociación”. Una finalidad de la que, como es natural, se hace eco esta negociación colectiva. Y que atestigua la doctrina judicial'5.

\footnotetext{
${ }^{1}$ Objeto de comentario, entre otros, por SEMPERE NAVARRO, A.V., "Contratación como fijo de obra para varias edificaciones indeterminadas. Comentario a la STS de 30 de junio de 2005”, Aranzadi, Repertorio de Jurisprudencia, 2005, vol. VII, Estudios, núm. 46, págs. 21737 a 21747.

${ }^{2}$ Como con lucidez atestigua la doctrina. Véase al efecto ARAGÓN GÓMEZ, C., "El contrato fijo de obra en el nuevo convenio de la construcción, ¿̨un mecanismo de estabilidad en el empleo?”, Relaciones laborales, ISSN 0213-0556, núm. 1, 2008, apartado II.1.

${ }^{3}$ Párrafo que trata de acrisolar las reflexiones de RODRÍGUEZ ESCANCIANO, S., "Sostenibilidad laboral en el sector de la construcción: la estabilidad en el empleo como premisa", Temas Laborales, núm. 152, 2020, pp. 14 a 20. Entrecomillado, página 14. Destaca también la autora que este sector no constituía, a resultas del RD 463/2020, actividad interrumpida pero tampoco se declaraba esencial.

${ }^{4}$ LUJÁN ALCARÁZ, J., "El contrato fijo de obra del sector de la construcción en la reforma laboral de 2010”, Revista Doctrinal Aranzadi Social num.12/2010, epígrafe 4.

${ }^{5}$ La STSJ de Cataluña (Social) núm. 7284/1999, de 21 octubre, estima así, como botón de muestra, acrisolando las ideas expresadas, que esta modalidad contractual no es contraria "a las normas de derecho necesario contenidas en el art. 15 del Estatuto de los Trabajadores", subrayando, en relación con un convenio provincial pero plenamente extrapolable, que la finalidad es "flexibilizar de alguna forma la contratación de trabajadores en un sector tan singular como el de la construcción, suprimiendo la necesidad de formalizar diferentes contratos para cada una de las obras, y lo que es más importante, contando siempre con el consentimiento del
} 
Más allá de aquella precisión legal -y de las que citamos de inmediato-, y a su amparo, el régimen jurídico del contrato fijo de obra descansa esencialmente en la autonomía colectiva. Como se viene a reconocer en la DA tercera ET, "lo dispuesto en el artículo 15.1.a) y 5 y en el artículo 49.1.c) se entiende sin perjuicio de lo que se establece o pueda establecerse sobre la regulación del contrato fijo de obra, incluida su indemnización por cese, en la negociación colectiva de conformidad con la disposición adicional tercera de aquella Ley 32/2006". DA tercera ET que sucede a la DA primera, número 2, de la Ley 35/2010, de rúbrica "Negociación colectiva y modalidades contractuales", y que prácticamente reproduce su contenido, aludiendo ya expresamente a la figura a estudio.

$Y$, en efecto, la negociación colectiva a distintos niveles ha prestado una gran atención a esta figura. Centraremos, no obstante, este análisis en la negociación colectiva sectorial estatal, aquella invitada por la LSC a adaptar el contrato de obra o servicio determinado en este sector. Regulación convencional al decir de autorizada doctrina "muy elaborada", que regula en detalle las condiciones de trabajo del sector a la par que "articula y estructura la negociación colectiva de ámbitos geográficos inferiores (provinciales o, en su caso, de Comunidad Autónoma)" y "produce una gran homogeneización de las condiciones de trabajo"6.

Por supuesto, el hecho de centrarnos en la negociación colectiva sectorial estatal no prejuzga la trascendencia, ni mucho menos obvia la existencia, de previsiones en la negociación colectiva, particularmente sectorial, a un nivel inferior. Negociación que, atendiendo a la doctrina judicial, podría incluir diferencias relevantes en algún aspecto ${ }^{7}$.

Pues bien, aquella negociación colectiva sectorial estatal acoge, desde su primera versión, este contrato. Destinando a regular el mismo un régimen jurídico apenas disperso en su seno. De hecho, se vertebra desde el claro protagonismo de una específica atención en un artículo dedicado al mismo. Y, en consecuencia, intitulado bajo su denominación ${ }^{8}$. Conjuntamente con este específico tratamiento, esta sucesión de convenios contempla el contrato fijo de obra en un número mínimo de otros preceptos. Preceptos cuyo régimen jurídico no tiene el mismo por objeto principal. En este caso, la continuidad no cubre la totalidad de las referencias convencionales advertidas.

\footnotetext{
trabajador cuya oposición impediría al empresario ejercitar esta posibilidad". Afirma también que con ello "se facilita una mayor estabilidad en el empleo de los trabajadores; imponiendo en todo caso como cautelas especiales la limitación a tres años, y como ya hemos dicho, la imprescindible conformidad del propio trabajador”. En idéntico sentido, la STSJ de Andalucía, Granada (Social, Sección 1a) núm. 1854/2017, de 8 septiembre, manifiesta que "se está en presencia de una modalidad contractual específica para las obras en construcción que tuvo como finalidad acabar con la precariedad laboral en el indicado sector, lo que le hace ser diferente en su estructura y contenido del resto de contratos temporales".

${ }^{6}$ RODRÍGUEZ ESCANCIANO, S., "Sostenibilidad laboral en el...”, pp. 27 y 28.

${ }^{7}$ La STSJ de Navarra (Social, Sección 1a) núm. 100/2006, de 24 abril, como botón de muestra, manifiesta que existe en ocasiones una disparidad de criterios en las Salas de lo Social de los Tribunales Superiores de Justicia sobre aspectos de este contrato "propiciada en la mayoría de los casos por los dispuesto en los Convenios Colectivos Provinciales".

${ }^{8}$ Concretamente, en los siguientes artículos nucleares: 8 del Acuerdo estatal sectorial de 1989 para el sector de la construcción, cuya publicación se ordena mediante Resolución de la DGT, de 7 de marzo de 1989 (BOE, 22.III; AESC-1989); 8 del Acuerdo Estatal Sectorial de la Construcción para 1990, cuya publicación se ordena mediante Resolución de la DGT, de 1 de marzo de 1990 (BOE, 13-III; en adelante, AESC-1990); 29 del Convenio Colectivo General del Sector de la Construcción de 10 de abril de 1992, cuya publicación se ordena mediante Resolución de la DGT de 4 de mayo de 1992 (BOE, 20-V, en adelante CGSC-I); 28 del Convenio Colectivo General del Sector de la Construcción, publicación ordenada mediante Resolución de la DGT, de 30 de abril de 1998 (BOE, 4-VI; en adelante, CGSC-II); 28 del Convenio Colectivo General del Sector de la Construcción 2002-2006, cuya publicación se ordena mediante Resolución de la DGT, de 26 de julio de 2002 (BOE, 10-VIII; CGSC-III); 20 del IV Convenio Colectivo General del Sector de la Construcción, publicada mediante Resolución de la DGT, de 1 de agosto de 2007 (BOE, 17-VIII; CGSC-IV); 24 del V Convenio Colectivo del Sector de la Construcción, cuya publicación se ordena por Resolución de 28 de febrero de 2012 (BOE, $15-$ III; CGSC-V); y 24 del vigente VI Convenio Colectivo General del Sector de la Construcción (Resolución de 21 de septiembre 2017, BOE, 26-IX; VI CGSC). Convenios que, desde el CGSC-V, incluyen una precisión normativa expresa para excepcionar de su ámbito temporal general algunas materias, entre las que se incluye el precepto que específicamente regula esta figura. Esto es, el contrato fijo de obra, cuya regulación tiene una vigencia inicial de 5 años (art. 7 en ambos casos, CGSC-V y VI CGSC).
} 


\section{Análisis del régimen jurídico de la negociación colectiva sectorial estatal y de su evolución}

En una aproximación general, el AESC-1990 introduce ciertas novedades en relación con su precedente AESC-1989, observándose desde entonces una clara línea de continuidad, con matices menores, hasta el IV-CGSC del año 2007. Éste rompe esa continuidad -la de los artículos 8 AESC1989, 8 AESC-1990, 28 CGSC-II y 28 CGSC-III-, incluyendo importantes modificaciones. Abre, en definitiva, una nueva y segunda línea de continuidad que llega hasta nuestros días, con el actualmente vigente VI CGSC, del ańo 2017. Convenio que sólo incluye las modificaciones necesarias para actualizar las referencias legales a la nueva versión del ET del año 2015. Una constante que comprende los artículos 20 CGSC-IV, 24 CGSC-V y 24 VI CGSC. Constante que desde el año 2007 incorpora una cierta contextualización del contrato incrementando claramente las referencias legales a la legislación estatal, en concreto al ET y a la LSC.

Adentrándonos en su análisis, las materias que han sido tradicionalmente objeto de tratamiento son las que a continuación se abordan.

\subsection{Formalización escrita del contrato y periodo de prueba}

En primer lugar, su formalización "siempre por escrito" (art.8 AESC-1989, art. 8 AESC-1990, 28 CGSC-II, art. 20 CGSC-IV, art. 24 CGSC-V y art. 24 VI CGSC). Recuerda la STS (Social) de 17 marzo 1980 (RJ 1980\1422) que el art. 42 de la Ordenanza Laboral de la Construcción establecía ya que los contratos fijos de obra "han de constar por escrito y - a la sazón- estar visados por el Sindicato".

Asistemáticamente, fuera de aquel precepto específico sobre el contrato fijo de obra, se observa desde el CGSC-II la exención de periodo de prueba para estos contratos de constar en la cartilla profesional la acreditación de su cumplimiento en cualquier empresa anterior (arts. 25 CGSC-II; 25 CGSC-III; 17 CGSC-IV, que opera el cambio de la referencia a la cartilla profesional por la titularidad de la tarjeta profesional de la construcción; 21 CGSC-V; y 21 VI CGSC).

\subsection{Su, controvertida, naturaleza como modalización del contrato de obra o servicio determinado}

En segundo lugar, la identificación del contrato a través de la delimitación de su objeto, "la realización de una obra o trabajo determinados" (art.8 AESC-1989, art. 8 AESC-1990, 28 CGSC-II y 28 CGSC-III, que sólo añade una referencia al art. 15.1 a). Cuestión ésta que naturalmente se conecta con el apartado destinado al estudio de su duración.

Con posterioridad a aquel CGSC-III, la citada STS (Social) de 30 junio 2005, advierte que, en efecto, «no se diseńa un nuevo contrato temporal al margen del art. $15 \mathrm{ET}$, lo que, por lo demás, no podría hacer válidamente pues, pese a su condición normativa y a su carácter vinculante, el Convenio está obligado a respetar las normas legales de carácter mínimo necesario, y el art. 15 lo es. Trata, simplemente, de evitar las cargas burocráticas que supondría la formalización de sucesivos contratos con un mismo trabajador, para "ajustarse mejor a las necesidades productivas de la empresa, a la vez que facilitar una mayor estabilidad en el empleo de los trabajadores"».

Retomando la sucesión de convenios, el art. 20 CGSC-IV ancla el contrato en la -entonces- nueva disposición adicional tercera de la Ley 32/2006, haciendo valer que ésta "otorga la negociación colectiva de ámbito estatal la facultad de adaptar al sector de la construcción el contrato de obra o servicio determinado regulado con carácter general en el artículo 15 del Estatuto de los 
Trabajadores". Contrato que, advierte dicho art. 20, es en especial una adaptación del art. 49.1. c) y del art.15.5 y $1 \mathrm{a}$ ), que con independencia de la duración del contrato fijo de obra no será de aplicación en su párrafo primero, continuando manteniendo los trabajadores la condición de "fijos de obra", "tanto en estos casos como en los supuestos de sucesión empresarial del artículo 44 E.T. o de subrogación regulado en el Artículo 23 del presente Convenio General”.

En idéntica línea a la del CGSC-IV, el art. 24 CGSC-V, añade a estas referencias a la legislación pública estatal la correspondiente a la DA primera, apartado 2, de la Ley 35/2010, aprobada tras el CGSC-IV, recordando también que otorga "a la negociación colectiva de ámbito estatal la facultad de adaptar al sector de la construcción el contrato de obra o servicio determinado regulado con carácter general en el artículo 15 del E.T.”. Línea que igualmente mantiene el actual art. 24 CGSC VI, que cita como legislación estatal de cobertura de esta adaptación la DA tercera del R.DLeg. 2/2015 y la Ley 32/2006, omitiendo la DA primera, apartado 2, Ley 35/2010, derogada por la D.D. única 4 R.D-Leg. 2/2015.

Citar, como colofón de este apartado, la STSJ de Madrid (Social) núm. 637/2019, de 7 junio. Pronunciamiento que recuerda que este contrato también se aplica cuando la actividad para la que se contrata es un servicio si "esta actividad está incluida en el ámbito funcional de la norma convencional de referencia". Y, "por tanto, se le aplican igualmente las previsiones en materia de contrato fijo de obra contenidas en su artículo 24".

\subsection{Duración del contrato}

En el AESC-1989 -que expresa entre otros propósitos el de recuperar este contrato fijo de obra- la duración del contrato será, con carácter general, "la de los trabajos del oficio y categoría del trabajador en la obra para la que haya sido contratado". El AESC-1990 precisa que el contrato terminará cuando concluyan estos trabajos y ańade, con ese mismo carácter general, que "el contrato es para una sola obra, con independencia de su duración”.

Sentada aquella regla supuestamente general, el AESC-1989 incorporaba ya la singularidad que, en nuestra opinión, da carta de naturaleza a la existencia de este contrato fijo de obra modalización del contrato por obra o servicio determinado. Y que ha perdurado hasta nuestros días. Esto es, que este personal "previo acuerdo de las partes (...) podrá prestar servicios a una misma Empresa y en distintos Centros de trabajo de una misma provincia durante un período máximo de tres ańos consecutivos sin perder dicha condición y devengando los conceptos compensatorios que correspondan". El AESC-1990 precisa los conceptos compensatorios que correspondan "por sus desplazamientos". Y el art. 28 CGSC-III añadiría que a tal efecto "suscribirán el correspondiente documento según el modelo que figura en el anexo IV", incorporando naturalmente dicho anexo.

Cumplido ese periodo de tres años, añade el AESC-1990 que "la Empresa podrá ofrecer al trabajador la continuidad en la misma como fijo de plantilla", cesando en caso contrario "automáticamente en la Empresa". En tal caso la empresa deberá "comunicar por escrito el cese al trabajador antes de cumplirse el período máximo de tres años", adquiriendo el trabajador "la condición de fijo de plantilla" si se cumple este periodo sin dicha comunicación. Periodo de tres años que, por cierto, era de dos en la antigua Ordenanza.

Por su parte, el posterior art. 28 CGSC-II sigue la línea del AESC-1990 pero incorpora la necesidad de que el acuerdo sea "expreso" y, además, junto a los matices ya reseńados, una precisión sustantiva de gran importancia, también mantenida hasta nuestros días. Y es que esa duración máxima de tres ańos se excepciona cuando los trabajos de la especialidad del trabajador fijo de obra "en la última obra se prolonguen más allá de dicho término". 
El CGSC-III, en su anexo IV, así como, al menos, el vigente VI CGSC, en su anexo II, incluyen, en relación con esta cuestión, un modelo de renovación del contrato fijo de obra.

El art. 20 CGSC-IV mantiene esta posibilidad de prestar servicios en una misma empresa y en distintos centros, aclarando, lo cual tiene una extraordinaria relevancia a efectos de reforzar la exclusión de la aplicación del art. 15.5 ET por cambio de obra dentro de un mismo contrato, que se produce "manteniéndose el carácter de único contrato". Incluye también la afirmación de que "en este supuesto y con independencia de la duración total de la prestación, tampoco será de aplicación lo establecido tanto en el apartado 1.a) párrafo primero del artículo 15 E.T. como en el apartado 5, continuando manteniendo los trabajadores, como se ha indicado, la condición de "fijos de obra" ". Y con esta letra pasó al art. 24 CGSC-V hasta llegar al actual art. 24 VI CGSC.

La traída STS (Social) de 30 de junio de 2005 hace hincapié en los requisitos de esta singularidad, esencial a la figura a estudio. En primer lugar, la observancia de los propios legales subrayados por la reiterada STS (Social) de 30 junio 2005 en relación con el contrato de obra o servicio determinado: "a) que la obra o servicio que constituya su objeto, presente autonomía y sustantividad propia dentro de lo que es la actividad laboral de la empresa; y b) que se especifique e identifique en el contrato, con precisión y claridad, la obra o el servicio que constituye su objeto". Criterio anticipado y seguido por la doctrina de suplicación?. Esta identificación precisa de la obra no se da cuando se designación genéricamente dos poblaciones en que se ejecuta (SJS núm. 1 de Valencia núm. 106/2012, de 29 febrero)

En segundo lugar, el requisito consistente en el "acuerdo expreso para cada uno de los distintos centros sucesivos", que "constituye un mandato que "solo se puede cumplir, válidamente, de dos modos. O reseñando en el propio contrato todas y cada una de las obras en las que se va a prestar servicios con expresa aceptación del trabajador, o bien suscribiendo acuerdos posteriores con igual y plena concreción antes de iniciar el trabajo en cada nueva obra. Modalidad esta última que, por cierto, recoge ya el actual Convenio General de la Construcción -CGSC-III-, incluyendo en su Anexo IV"». No siendo "posible sustituir válidamente el acuerdo expreso para cada uno de los distintos centros de trabajo sucesivos que exige el Convenio con respeto de las previsiones legales, por una incondicional aceptación del trabajador de una cláusula impuesta por la empresa en el momento mismo de la contratación, en que es más evidente la posición de desigualdad de las partes, para trabajar en unas obras cuya ubicación, calidad y tipo de actividad ignora» [STS (Social) de 30 junio 2005].

La STSJ de Islas Canarias, Las Palmas (Sala de lo Social, Sección 1a) núm. 862/2004, de 20 septiembre había anticipado -en aplicación del CGSC-III amén del provincial- ese criterio, afirmando que para poder articular el contrato fijo de obra en distintas obras "las mismas han de ser especificadas en el contrato o, en su caso, en un pacto posterior que ha de ser expreso y escrito, con especificación de las nuevas obras", negando "validez a las cláusulas que hacen una alusión genérica a los preceptos citados, por entender que ello supone la desnaturalización del contrato de obra, al desconocerse las obras para las que se contrata al trabajador y, por tanto, cuando será la finalización del contrato".

\footnotetext{
${ }^{9}$ En esta misma línea, recordando la STS de 30 de junio de 2005 antedicha, las SSTSJ de Galicia (Sala de lo Social, Sección 1ª) núm. núm. 6620/2015, de 30 noviembre, y 465/2016, de 29 enero, advierten que el contrato fijo de obra en el sector de la construcción "debe acomodarse a las normas legales y reglamentarias que regulan el contrato de obra y a la interpretación jurisprudencial que se acaba de transcribir en relación con los requisitos legales y reglamentarios del mismo". Y lo hacen en relación con un supuesto al que resulta aplicable el CGSC-V. Las sentencias estiman que se incumplen los requisitos legales porque, en primer lugar, identificar la obra como "Túnel de Prado" es insuficiente cuando hay dos obras independientes, para la vía derecha y para la vía izquierda, a pesar de alegar la empresa ser subcontratista únicamente para la obra de la vía derecha. También porque no se identifica la tercera obra y porque el tiempo excede de los tres años, entre otras razones como son, en la primera, la prestación indistinta de trabajo en las diversas obras.
} 
No es suficiente a efectos de este conocimiento, y acuerdo del trabajador, que la sucesión de contratos quede plasmada en las sucesivas notificaciones al INEM, en las que figuran los cambios en el centro de trabajo operados a lo largo de la relación laboral [STSJ de Asturias (Social, Sección 1a) núm. 2425/2010, de 8 octubre].

En tercer lugar, las SSTSJ de Galicia (Sala de lo Social, Sección 1a) núm. 6620/2015 y 465/2016, afirman que el art. 24 CGSC-V (cuyo tenor es idéntico al actual en este extremo, como en su casi práctica totalidad) «no permite en modo alguno la prestación de servicios simultánea en varias obras, aunque éstas sean obras a las que previamente fue destinado (cambiado) el trabajador con su consentimiento. Precisamente el sentido de la norma es claro al respecto, pues utiliza expresamente la palabra "sucesivos"».

Dejar constancia, no obstante, de que la doctrina de suplicación no es unívoca sobre el rigor con el que aplicar, en algunos extremos, estos requisitos legales. Siendo acaso el criterio a nuestro modo de ver más adecuado el advertido en la STSJ de Castilla-La Mancha (Social, Sección 2a) núm. 1046/2012, de 4 octubre, llamando a valorar la entidad de la desviación respecto de la literalidad legal. Admite así este pronunciamiento que el hecho de que el trabajador sea «ocupado "normalmente" en la realización de las obras que son objeto de contrato, (..) no implica que la realización de otras ocupaciones distintas a las expresamente pactadas, sea cual fuere su duración, pueda calificarse de fraudulenta, sino que habrá de estarse a cada caso concreto para valorar la duración e importancia de tal circunstancia». Y concluye la regularidad del contrato que abarca casi tres años, a pesar de advertir la prestación de servicios durante un total de 45 días en otras obras distintas de la empresa por razones urgentes y perentorias ${ }^{10}$.

Más allá de esta lectura judicial, la doctrina se encuentra dividida sobre esta particularidad, esencial al contrato fijo de fin de obra. Una parte ha refrendado la validez de este contrato fijo de obra considerando que, esta virtualidad del mismo, no produce una descausalización del contrato. Y subraya que "el papel de la negociación colectiva en esta materia es muy limitado pues nos hallamos frente a normas de derecho necesario indisponibles por tanto para la misma" ${ }^{11}$. No obstante, otra doctrina expone los cuestionamientos habidos a esta consideración como único contrato, recuperando la idea del Profesor Cruz Villalón de tratarse más bien de un "procedimiento de celebración simplificada de distintos y sucesivos contratos, si bien tratados como una sola relación jurídica», porque de ser un único contrato se estaría rompiendo la causalidad en la contratación temporal. Reflexiona también la doctrina sobre la idea de tratarse esa sucesión de obras como una novación extintiva y recuerda que la propia ITSS considera que estamos ante diferentes contratos ${ }^{12}$. En el apartado de conclusiones elevaré una primera reflexión propia al respecto.

\footnotetext{
${ }^{10}$ En esta línea, la STSJ de Madrid (Social, Sección 6a) de 4 junio 1997 (Recurso de Suplicación 4062/1996) estima que "se ha rebasado el plazo de los tres años en escasos días y en consecuencia, atendidas las circunstancias del caso, tal hecho carece de suficiente relevancia para atribuir al trabajador la cualidad de fijo". De destacar, además, por ser anterior a la modificación legal del CGSC-II, abriendo la posibilidad de prolongar la duración si se continuara en la última obra y esta se extendiese en el tiempo, al contrario de la STSJ de Islas Canarias, Las Palmas (Social, Sección 1a) núm. 213/2004, de 10 marzo, que entiende el actor ha devenido fijo de plantilla por exceder la duración máxima de este contrato contemplada en el CGSC 1997, de tres ańos. Requisitos que no se dejan de cumplir cuando se produce un cambio de la categoría profesional en el mismo puesto de trabajo, realizando las tareas de la previa y cobrando conforme a ello, ni por el hecho de integrarse su empresa en una UTE adjudicataria de la obra (STSJ de Cataluña (Social, Sección $1^{\text {a) }}$ núm. 7278/2014, de 4 noviembre).

${ }^{11}$ VICENTE PALACIO, Ma . A., "El contrato fijo de obra en el sector de la construcción: sobre la previsión convencional de destinar al trabajador a obras distintas de las inicialmente contratadas ubicadas dentro de la misma provincia y por una duración máxima de tres años consecutivos. (Comentario a la STSJ Cataluña 21 octubre 1999)", Aranzadi Social, ISSN 1131-5369, núm. 3, 1999, págs. 2961-2970. Debido es destacar la aportación monográfica de esta misma autora El contrato temporal para obra o servicio determinado, Tirant lo Blanch, Valencia, 1996, 455 páginas.

${ }^{12}$ ARAGÓN GÓMEZ, C., "El contrato fijo de obra...”, apartado III.
} 


\subsection{La inaplicación de ciertas garantías legales}

En cuarto lugar, y en íntima conexión con lo anterior, este precepto específico regulador de la figura a estudio contempla la previsión de inaplicación de ciertas garantías legales. Las contempladas en el art. 15.5 ET y otras.

El art. 20 CGSC-IV incluía unos números, 4 y 5, señalando que "teniendo en cuenta la especial configuración del sector de la construcción y sus necesidades, sobre todo en cuanto a la flexibilidad en la contratación y la estabilidad en el empleo del sector mejorando la seguridad y salud en el trabajo así como la formación de los trabajadores -añadiendo en este punto el art. 24 CGSC-V una referencia a la DA primera 2 Ley 35/2010 que se sustituye por la DA tercera ET 2015 en el art. 24 VI CGSC-, no se producirá sucesión de contratos por la concertación de diversos contratos fijos de obra para diferentes puestos de trabajo en el sector, teniendo en cuenta la definición de puesto de trabajo dada en el artículo 18 del presente Convenio, y por tanto no será de aplicación lo dispuesto en el párrafo 5..$^{\circ}$ del artículo 15 del Estatuto de los Trabajadores”.

Una llamada al papel de esta singular figura contractual como fortalecedora de la estabilidad en el empleo que, desde el CGSC-IV, se tiene presente en esta negociación colectiva sectorial estatal a la hora de regular la jubilación obligatoria. Objetivo de mejora de la estabilidad en el empleo cuya plasmación en estos convenios colectivos se afirma encontrar, entre otros aspectos que se identifican, en la regulación de este contrato fijo de obra del sector (art. 93 CGSC-IV; 97 CGSC-V; y 96 VI-CGSC ${ }^{13}$ ). Que, en consecuencia, no es la única previsión convencional que se manifiesta servir a tal propósito.

En contra de esta declaración de nuestra negociación colectiva, cualificada doctrina sostiene, sin embargo, que "el contrato fijo de obra - a diferencia de lo que supuso tradicionalmente- ya no es un mecanismo que favorezca la estabilidad en el empleo en el sector de la construcción, por lo que su utilización difícilmente puede justificar, por sí misma, la limitación de la vida activa de los trabajadores del sector, mediante el establecimiento de edades máximas de jubilación" ${ }^{\prime 4}$.

El art. 20.5 CGSC-IV -al igual que el art. 24 CGSC-V y vigente art. 24 VI CGSC-proseguía afirmando que "por lo tanto la contratación, con o sin solución de continuidad, para diferente puesto de trabajo mediante dos o más contratos fijos de obra con la misma empresa o grupo de empresas en el período y durante el plazo establecido en el artículo 15.5 E.T., no comportará la adquisición de la condición establecida en dicho precepto" ${ }^{15}$. Señalando que "a tal efecto nos encontramos ante puestos de trabajo diferentes cuando se produce la modificación en alguno de los factores determinados en el artículo 18 del presente Convenio" (referencia al art. 18 sustituida por el art. 22 en el art. 24 CGSC-V, así como en el art. 24 VI CGSC). Y ańadiendo que "la indicada adquisición de condición tampoco operará en el supuesto de producirse bien la sucesión empresarial establecida en el artículo 44 E.T. ${ }^{16}$ o la subrogación recogida en el artículo 23 del presente Convenio" (referencia al art. 23 sustituida por el art. 27 en el art. 24 CGSC-V, así como en el art. 24 VI CGSC).

\footnotetext{
${ }^{13}$ Art 96 objeto de modificación en reunión cuya acta se ordena publicar mediante Resolución de 23 de abril de 2019 (BOE, 26-IV) sin afectar la referencia a este contrato. Lo mismo sucede con el art. 7 modificado sin afectar al mismo. Algo que se repite, en relación con este artículo 7 de este mismo VI CGSC, con la modificación cuya publicación ordena la Resolución DGT de 23 de octubre 2019 (BOE, 5-XI).

${ }^{14}$ ARAGÓN GÓMEZ, C., "El contrato fijo de obra...”, apartado IV. Doctrina que analiza, precisamente, el CGSC-IV.

${ }^{15}$ Un sistemático esfuerzo expositivo de argumentos a favor y en contra de la aplicación del art. 15.5 ET al contrato fijo de obra cabe apreciar en NICOLAS BERNAD, J.A., "Consideraciones sobre el contrato fijo de obra en el nuevo Convenio General del Sector de la Construcción para los años 2007-2011”, Actualidad Jurídica Aranzadi, num.748/2008.

${ }^{16}$ En relación con la aplicación del art. 44 ET, la SJS número 1 de Ceuta núm. 181/2019, de 27 agosto, estima que la sucesión de una UTE en el servicio de mantenimiento de la seńalización del Puerto de Ceuta, en cuya prestación cesa la empresa que contrata con el trabajador, determina la correcta extinción contractual. Advierte así la Sentencia que esto es así porque no constituye un supuesto del art. $44 \mathrm{ET}$ por desconocer si concurren los requisitos legales, ni haber sido alegado por las partes. Tampoco cabe deducirlo de la
} 
Régimen convencional del que se hacen eco nuestros Juzgados y Tribunales. Las SSTS (Social), de 25 de mayo de 2011 (R.Ud. 1907/2010) y de 15 de junio de 2011 (Rec. Ud. 2005/2010), ambas sobre el tenor de este precepto con anterioridad a la Ley $35 / 2010^{17}$, entienden así que no procede la aplicación del art. 15.5 ET y, en consecuencia, tampoco la consiguiente adquisición de la condición de fijo, cuando se trata de este contrato fijo de obra. Siempre, eso sí, que no exceda de tres ańos. Y ello a pesar de que se encadenen varios de estos contratos, afirmando existir diversidad de puesto de trabajo conforme al convenio colectivo aplicable. Cierto que a la letra actual del precepto esta identidad o cambio del puesto de trabajo parece resultarle indistinto.

Cuestión ésta, la identificación del puesto de trabajo, que ha generado división judicial, justificando la emisión de votos particulares alejados de la aplicación de la definición ofrecida por el convenio. Votos que, como hace la propia doctrina, diseccionan los elementos locativo y funcional propios de aquél, y analizan la falta de homogeneidad en el uso de este concepto. Subrayando en ocasiones la doctrina el carácter decisivo del elemento funcional.

\subsection{Consecuencias de la paralización temporal y extinción contractual}

En quinto lugar, estos preceptos contemplan las consecuencias de la paralización temporal de una obra. El AESC-1989 advertía ya que el contrato se "verá afectado por cualquier suspensión o paralización temporal que pueda producirse en la obra, extinguiéndose por este motivo dicho contrato y comprometiéndose el empresario a emplear de nuevo al mismo trabajador cuando las causas de suspensión o paralización de la obra hubiesen desaparecido. Dicho contrato se entenderá finalizado cuando estos supuestos se conviertan en definitivos".

Con mucha mayor extensión, y orillando algunos cambios en la redacción a nuestro parecer menores, el AESC-1990 se refiere únicamente a la paralización temporal de una obra, excluyendo la debida a conflicto laboral, y ańadiendo que ha de ser motivada por "causa imprevisible para el empresario principal y ajena a su voluntad". Omite, pues, la referencia a la suspensión. Y ordena el procedimiento a seguir, de modo que tras dar cuenta la empresa a la "representación de los trabajadores del centro o, en su defecto, a la comisión paritaria provincial, operarán la terminación de

\footnotetext{
legislación administrativa ni de los pliegos, que no hacen referencia a las consecuencias laborales, de la sucesión empresarial. Y añade que tampoco se trata de una sucesión convencional, toda vez que la cesión o sustitución del empresario no se encuentra regulada en el Convenio de aplicación. Cita además la STS de 20 de marzo de 2015 como fundamento para afirmar que "en aquellos casos en los que se renueva la causa que justificó el contrato temporal, como ocurre en este caso, el contrato temporal se mantiene, toda vez que la "obra o servicio" para el que fue contratado sigue vigente. Que no resulta de aplicación en el ámbito de la construcción los límites indicados en el artículo 15.1 del ET. La única consecuencia es entender que la relación laboral entre las partes, seguía siendo temporal, no adquiriendo el carácter indefinido".

${ }^{17}$ Ambas con voto particular que, en el primer caso, cuestiona el tratamiento de cada cambio de centro de trabajo como si se tratare de una relación ex novo siendo así que no ha de ser considerado como tal en el seno del contrato de fijo de obra, llegando a manifestar que "con el debido respeto a la Sala, entiendo que la solución alcanzada conduce al absurdo: acaba por afirmarse que cada obra es autónoma e independiente del historial inmediato anterior en un convenio que, precisamente, permite que la contratación para una obra admita el cambio de obra". En consecuencia, tratándose de un mismo contrato y puesto de trabajo habría de operar la tutela y consiguiente sanción del art. 15.5 ET. En la segunda de las citadas Sentencias el voto particular se construye sobre la base de que la habilitación a la negociación colectiva conferida por el precepto en cuestión "no es para excepcionar o limitar la aplicación de la citada norma, sino muy contrariamente para adoptar las previsiones -ajustadas al sector y al "puesto de trabajo»- que sean necesarias en orden a evitar la utilización abusiva de la contratación temporal" y que la conclusión alcanzada por la sala llegaría al "punto de consentir-conforme a sus previsiones y al planteamiento de la sentencia recurrida- una situación de temporalidad que persistiría sin solución de continuidad durante los doce años del supuesto de autos, lo que en la práctica equivaldría a inaplicar -que no matizaren el sector de la construcción toda la normativa legal de que tratamos". Parte también de cuestionar el concepto de puesto de trabajo asumido sobre la base de que nace de una combinación de aspectos funcionales y locativos que en el caso en cuestión no conllevan nuevo puesto de trabajo. Aproximación al concepto de puesto de trabajo que ulteriormente se aprecia en la STSJ de Asturias (Social, Sección 1a) núm. 2969/2011, de 25 noviembre. Votos particulares no inhabituales en estos litigios, como se aprecia también en la STSJ de País Vasco, (Sala de lo Social, Sección 1a) núm. 896/2010, de 30 marzo.
} 
obra y cese previstos en el supuesto primero". Representación de los trabajadores del centro o, en su defecto, comisión paritaria que "dispondrá, en su caso, de un plazo máximo improrrogable de una semana para su constatación, a contar desde la notificación”. Añade, además, que "previo acuerdo entre las partes, el personal afectado por esta terminación de obra podrá incluirse en lo regulado para el supuesto segundo". Esto es, pasar a prestar servicios en otro centro de trabajo de la empresa en la provincia. El art. 28 CGSC-II reproduce en este ámbito lo contemplado en el AESC-1990. Y esta es la línea seguida en lo sucesivo, incluido el art. 24 del actual VI CGSC.

Cabe indicar que, al margen de este nuclear precepto y reconociendo la especialidad del mismo, la negociación colectiva tiene presente la especificidad de este contrato al regular la suspensión contractual por causa de fuerza mayor temporal. Concretamente al enumerar entre las situaciones determinantes de esta situación la "paralización de la obra o parte de ésta, por orden gubernativa, resolución administrativa u otras causas similares ajenas a la voluntad del empresario, sin perjuicio de lo establecido, al respecto, para el contrato fijo de obra, en el presente Convenio" (arts. 94 CGSC-I, 93CGSC-II, 95 CGSC-III, 87 CGSC-IV, 91 CGSC-V y 90 VI CGSC).

Una fuerza mayor afectada por la pandemia desde múltiples enfoques. Sin ánimo de exhaustividad, en primer lugar, por la incidencia del permiso retribuido recuperable contemplado en el RD-Ley 10/2020, de 29 de marzo (BOE, de idéntica fecha) en la previsión convencional de recuperación del 70 por 100 de las horas no trabajadas por interrupción de la actividad debida a causas de fuerza mayor. En segundo lugar, por repercutir en la regulación de supuestos que de esta institución efectúa el art. 90 VI CGSC. Por último, debido al nuevo concepto de fuerza mayor fruto de la pandemia sanitaria observable en el art. 22 Real Decreto Ley 8/2020, de 17 de marzo (BOE, 18-III) ${ }^{18}$.

Pandemia aparte, cualificada doctrina entendió que ésta, la suspensión, habría de ser en todo caso la solución a adoptar si la paralización es temporal, como supuesto de fuerza mayor. Fuerza mayor que motivaría la extinción por idéntica causa, encuadrable en los artículos 49.1 y 51 ET, sólo de ser la paralización permanente. Aplicando entonces, consecuentemente, la indemnización prevista en este precepto legal ${ }^{19}$. Y así cabría interpretar la referencia convencional recién mentada, considerando que la específica mención al contrato a estudio (el "sin perjuicio") lo es a otros efectos.

Centrados en la paralización temporal, cierto es que una circunstancia no definitiva difícilmente conlleva en su naturaleza la extinción -aunque una incapacidad calificada como permanente puede excepcionalmente conllevar la suspensión, si bien por su revisión-. Por otra parte, las garantías convencionales contempladas para esta situación en su consideración como cese parecen superiores a las de la suspensión. Se suman, en efecto, de una parte, la obligación empresarial de ofrecer un nuevo empleo al trabajador cuando las causas de paralización de la obra hubieran desaparecido", obligación que "se entenderá extinguida cuando la paralización se convierta en definitiva". Se suma, de otra parte, la previsión de extender la indemnización por extinción contractual a todos los supuestos anteriores, es decir, también a esta situación resultante de la paralización temporal. Previsión que entendemos el convenio anuda a la consideración de esta paralización temporal como causa de extinción.

Lo cierto es que el tratamiento jurídico parece responder a una doble consideración jurídica, aunando posiblemente su naturaleza última suspensiva, y consiguiente obligación empresarial modulada, llamémosla de reactivación laboral (similar mutatis mutandi a una reserva de un puesto equivalente), con los efectos propios de una extinción contractual, a lo que se entendería responde aquella coletilla legal: "sin perjuicio de lo establecido, al respecto, para el contrato fijo de obra, en el presente Convenio". Por lo demás, cualquier distanciamiento en su tratamiento respecto de la regla

\footnotetext{
${ }^{18}$ RODRÍGUEZ ESCANCIANO, S., "Sostenibilidad laboral en el...”, pp. 18 y 19.

${ }^{19}$ ARAGÓN GÓMEZ, C., “El contrato fijo de obra...”, apartado II.2.D.a.
} 
general del ET, en lo que esta tiene de imperativo cumplimiento, encuentra siempre en el camino de su justificación el amparo legal, siempre en su dimensión real, de la LSC y del ET -en las disposiciones citadas-, dado su carácter de ley especial.

La normativa convencional a estudio contempla, por último, la extinción contractual más allá de esta paralización. El art. 8 AESC-1989 establecía que esta extinción se produce "cuando la realización paulatina de las correspondientes unidades de obra hagan innecesario el número de los contratados para su ejecución, debiendo reducirse éste de acuerdo con la disminución real del volumen de obra realizada”. Recuerda la STS (Social) de 13 noviembre 1987 (RJ 1987\7869) que la doctrina reiterada de la Sala interpretaba el art. 44.c) de la Ordenanza de Trabajo en la Construcción en el sentido de entender que "el cese del personal contratado con carácter de fijo para la obra de que se trate, puede y debe producirse atemperadamente a la marcha y necesidades del personal adscrito a la propia obra y, por supuesto, en relación con las diversas especialidades, como matiza la norma”.

Esta extinción paulatina genera la duda, como bien apunta la doctrina, de cuál es el orden a seguir. Resulta natural, en primer lugar, atender al convenio o convenios de aplicación para advertir si se precisa éste ${ }^{20}$. En caso contrario, cualificada doctrina apunta que bastaría un "criterio racional cualquiera" ${ }^{\prime 2}$. Atender las clásicas prioridades -visibles en el Estatuto de los Trabajadores en otras materias- en razón de la antigüedad, representación que se ostenta, razones de cargas familiares, etc., constituye un buen anclaje para construir el mismo.

Cese que deberá "comunicarse por escrito al trabajador con una antelación de quince días naturales", siendo sustituible "este preaviso por una indemnización equivalente a la cantidad correspondiente a los días de preaviso omitidos calculada sobre el salario base del convenio, todo ello sin perjuicio de la notificación escrita del cese". A la hora de cuantificar la indemnización por cese, sin embargo, no se refería a ese salario base, sino que la cifraba en el " 4,5 por 100 , calculada sobre los conceptos salariales de las tablas del Convenio devengados durante la vigencia del contrato". Indemnización que "deberá incluirse en el recibo de salarios con la liquidación correspondiente al cese".

El art. 8 AESC-1990 sustituyó en el cálculo de esta indemnización aquella primera referencia al salario base del convenio por, en todo caso -también para la inobservancia del preaviso-, "los conceptos salariales de las tablas del Convenio". Redacción mantenida en el CGSC-II, añadiendo el CGSC-III que esto es así según lo previsto en el artículo 49.1.c) ET.

La STSJ de País Vasco (Sala de lo Social, Sección 1a), de 15 noviembre 2005 (Recurso de Suplicación 1641/2005) afirma que el deber de preaviso, y consiguiente indemnización en caso de incumplimiento, comprende los contratos de obra en los que se ha pactado una fecha cierta de terminación, con independencia de que el contrato alcance el año de duración contemplado en el régimen general del art. 49.1.c) ET. Esto es, considera que el carácter incierto de la duración de la obra o, por el contrario, la fijación de una fecha cierta, no son determinantes de la aplicación de esta previsión convencional.

CGSC-II y CGSC-III compartían el hecho de excepcionar expresamente -al margen del precepto central en la materia- de las indemnizaciones contempladas por extinción por cumplimiento del término esta modalidad contractual. Excepción que venía motivada por contemplar una indemnización específica en el apartado regulador de la figura (arts. 29 CGSC-II, en relación con contratos formalizados conforme a los entonces vigentes RD 2546/1994 o al RD 1989/1984; y 29 CGSC-III, en relación a contratos formalizados conforme al Real Decreto 2720/1998).

Esta referencia convencional, la expresa excepción, desaparece en el CGSC-IV hasta nuestros días. Tampoco está presente, en consecuencia, en el convenio actualmente vigente. Al contrario,

\footnotetext{
${ }^{20}$ ARAGÓN GÓMEZ, C., "El contrato fijo de obra...", apartado II.2.D.a.

${ }^{21}$ BARREIRO GONZÁLEZ, G. y FERNÁNDEZ DOMÍNGUEZ, J.J., "Contratación”, en VALDÉS DAL-RÉ, F. (Dir.), Comentarios al convenio general de la construcción, Fundación Anastasio de Gracia, Madrid, 1993, págs. 139 y 140.
} 
desde el año 2007 el enunciado convencional opta por advertir expresamente que, en cuanto a las causas y efectos de la extinción de este contrato, habrá de estarse, en concreto, a lo establecido en los artículos 49 a 57 ET y a las especialidades previstas en este Convenio (arts. 90 CGSC-IV; 94 CGSC-V; y 93 VI CGSC).

Más allá de la evolución en la redacción de este apartado, la extinción sigue, no obstante, contemplándose en similares términos a los de los convenios anteriores en los artículos 20 CGSC-IV y 24 CGSC-V y VI CGSC. Con dos salvedades más.

En primer lugar, la cuantía asciende al 7 por 100 desde el art. 20 CGSC-IV. Convenio que además incluye en su D.Ta $2^{\mathrm{a}}$ una previsión para los contratos preexistentes a su entrada en vigor. Contratos que mantienen la indemnización vigente al momento de su perfeccionamiento hasta la fecha de publicación de este IV Convenio, aplicándose desde entonces el nuevo porcentaje. Indemnización igualmente aplicable a la paralización temporal.

En segundo término, el art. 24 CGSC-V añade una referencia a la Ley 35/2010 que es sustituida por la propia al R.D.Leg. 2/2015 en el art. 24 VI CGSC. Convenio éste, vigente, que señala que se hará "siempre y en todo caso, respetando la cuantía establecida en el citado artículo $49.1 \mathrm{c}$ ) del E.T.", lo que parece difícil sea de otro modo pues ese 7 por 100 es superior a la cuantía de la indemnización contemplada en este precepto legal ${ }^{22}$.

Resulta no obstante insoslayable en esta materia, como en tantos otros aspectos, realizar un atento seguimiento, por una parte, de la aparentemente férrea voluntad política de modificar profundamente el régimen jurídico de la contratación temporal, contrato por obra o servicio determinado incluido. Y, por otra, del voluble criterio del TJUE en relación con la contratación temporal, con la Directiva 1999/70/CE del Consejo, de 28 de junio de 1999 (DOCE, 10-VII), y de este modo asegurar la acomodación de esta figura a la normativa de la UE ${ }^{23}$. Ya la STSJ de Andalucía, Granada (Social, Sección 1a) núm. 1854/2017, de 8 septiembre, se basó en la doctrina reflejada en las SSTS de 8 de junio de 2016 (Rec. 207/2015 y Rec. 112/2015) y en las numerosas sentencias del actual TJUE que cita, para desestimar la pretensión de una indemnización de 20 días de salario por ańo de servicio con límite de 12 mensualidades sustentada en el art. 53 ET por ser discriminatoria en relación con el art. $49 \mathrm{ET}^{24}$. Somos conscientes, no obstante, de que el TJUE se muestra particularmente pendular en sus pronunciamientos.

Seguimiento que, por cierto, también habría de proyectarse sobre la evolución de la doctrina abierta con la STS (Ud.) núm. 1.137/2020, de 29 de diciembre, en el ámbito de la descentralización productiva.

\footnotetext{
${ }^{22}$ Como expresamente la doctrina interpreta. Véase al efecto LUJÁN ALCARÁZ, J., "El contrato fijo de ...”, epígrafe 2.

${ }^{23}$ Una interpretación del TJUE que peca de desconocimiento sobre la naturaleza de algunas instituciones jurídicas en su diseño estatal (confundir extinción por cumplimiento del término -y consecuente cumplimiento del contrato- con una rescisión contractual, como botón de muestra), y consiguiente correspondencia con la normativa europea aplicable. Elocuente, en fin, es el título del comentario de DE LA PUEBLA PINILLA, A., «Principio y fin de la doctrina «de Diego Porras», o de cómo, en ocasiones, "el sueño de la tutela multinivel produce monstruos"», Revista de Información Laboral, num.7/2018.

${ }^{24} \mathrm{La}$ Sentencia recuerda que la aplicación directa del derecho originario europeo no es incondicional, sino que ha de generar obligaciones "precisas", "claras", "incondicionales" y "no requerir medidas complementarias". En cuanto a las Directivas, como "norma de resultado que es, contiene un mandato para alcanzar unos fines dirigido al Estado miembro de la Unión Europea, es decir, el receptor o sujeto pasivo de aquel mandato es el Estado, no el particular, por lo que resulta rechazable la aplicación de la directiva de forma horizontal entre particulares". Así, «las consecuencias negativas para la operatividad del Derecho de la Unión Europea que se derivan de la afirmada ineficacia directa horizontal de las Directivas sólo quedan parcialmente suplidas por la posible responsabilidad del Estado incumplidor por los daños y perjuicios que pueda causar su incumplimiento de la Directiva y por la eficacia interpretativa de las Directivas en la aplicación del Derecho nacional, conforme al principio de "interpretación conforme" ", sin forzar de forma indebida la normativa interna. Recordando que «la más reciente doctrina del Tribunal Europeo afirma que "el requisito de interpretación conforme no puede llevar a que una directiva cree, por sí misma e independientemente de una ley interna de adaptación, obligaciones para los particulares"» y que «la obligación del juez nacional de utilizar como referencia el contenido de una directiva cuando interpreta y aplica las normas pertinentes del Derecho interno... no puede servir de base para una interpretación contra legem del Derecho nacional».
} 
Llama la atención, además, aquella relevante Sentencia del TSJ de Andalucía, sobre el hecho de que el régimen indemnizatorio de este contrato se sustenta sobre un solo parámetro "a diferencia del resto de contratos de naturaleza temporal con derecho a la indemnización por extinción al tiempo de su terminación, donde el montante indemnizatorio se sustenta sobre dos parámetros: número de años de servicio y salario último devengado" (...) "de lo que se desprende que, estamos en presencia de parámetros indemnizatorios no equiparables, a efectos de declarar la existencia de razones objetivas que al margen de la mera naturaleza temporal entre los contratos de trabajo comparables, que justifican una distinta cuantificación en la indemnización".

La STSJ de Castilla y León, Valladolid (Social, Sección 1a), de 20 junio 2012, atribuye, para concluir, tal y como se desprende de las previsiones analizadas, la condición de indefinido al trabajador fijo de obra cuando su cese no responde a la causa especificada en la comunicación escrita, esto es, la finalización de la obra, al seguir trabajando -en el caso con labores de "repaso"- una vez que oficialmente ésta había sido rematada.

\section{Conclusión}

El contrato fijo de obra es una figura jurídica propia y tradicional en el ámbito de la construcción. Y que se caracteriza por erigirse como una relativa desnaturalización del contrato de fin de obra o servicio determinado. No por ser algo distinto a este, como subraya la señera STS (Social) de 30 junio 2005, sino por ser una vía para facilitar la formalización de un vínculo contractual estable en detrimento de lo que de otro modo serían sucesivos contratos. Más allá de la situación legal, que determina la norma y define la jurisprudencia, es lo cierto que probablemente existan, como dice Cruz Villalón, distintos contratos en el seno de una misma relación jurídica.

En los términos negociados, se trata, en nuestra opinión, de una versión "descausalizada” del contrato de obra o servicio determinado cuando comprende varias obras o servicios, posibilidad ésta abierta por la normativa a estudio y que ha constituido uno de los ejes de la discusión jurídica al efecto. Demandándose en tal caso la identificación precisa de la obra o servicio con autonomía y sustantividad propia, la necesidad de un acuerdo expreso en los términos legalmente requeridos o la razonable contención temporal e inexistencia de una prestación simultánea, más allá de la necesidad de analizar las circunstancias del caso concreto para ver si estas conllevan una desviación sustantiva de estos últimos requisitos legales.

Cierto es, por otra parte, que esta descausalización no debiera sorprendernos. También lo es, acaso en menor grado, la nacida en la legislación estatal, en el propio art. 15 ET, al establecer una concreta duración máxima de este contrato, puesto que lo que es verdaderamente causal en el mismo es sujetar su duración estrictamente, apegarla, a la propia de la obra o prestación de servicio que constituye su objeto. En la medida en que aquella o este demandan la prestación del trabajador. Cuanto más se desconozca esa ligazón natural menos se observa su causalidad. $\mathrm{O}$, como segundo ejemplo, el ultimísimo giro dado por nuestro TS tratando de adecuar su criterio al propio del TJUE en relación con las interinidades en las Administraciones Públicas. Una duración legal del contrato temporal formalmente garantista del trabajador, pero desvinculada de la sustantiva de la necesidad real de la prestación laboral, también erosiona la causalidad de la contratación temporal.

La cuestión estriba en consecuencia, al parecer, en si se descausaliza el contrato para tutelar al trabajador, en cuyo caso podría compadecerse con la tutela última del trabajador que la propia causalización de la contratación temporal persigue. O si esa descausalización no tutela al trabajador, en cuyo caso sería francamente cuestionable. Es decir, y en otras palabras, radica en verificar si esa versión flexibilizada de la duración de esta figura que, en definitiva, constituye su esencia y muy proba- 
blemente dé carta de naturaleza a la misma, contribuye o no a mejorar la calidad del empleo y, sobre todo, la estabilidad del mismo a que llama la DA tercera de la Ley 32/2006. De la que se hace eco la negociación colectiva, en particular la segunda gran línea de continuidad de la negociación colectiva sectorial estatal vista, la iniciada desde el año 2007. Y que reconoce la jurisprudencia de nuestro TS.

Es indudable que la exclusión en la aplicación del art. 15.5 ET -sobre la que acaso penda la espada de Damocles de la oscilante jurisprudencia del TJUE- y de otras garantías es un argumento de peso en contrario. Atribuimos, no obstante, un mayor peso en aras de la estabilidad laboral al tratamiento de la duración y de la extinción de esta figura, incluyendo la paralización temporal.

Respecto de esta última, cierto es que resulta difícil atribuir a una circunstancia no definitiva carácter extintivo de la relación laboral, probablemente porque no está en su naturaleza. Las garantías convencionales contempladas para esta situación en su consideración como cese parecen, sin embargo, superiores a las de la suspensión. Se suman, en efecto, la obligación empresarial de ofrecer "de nuevo un empleo al trabajador cuando las causas de paralización de la obra hubieran desaparecido" y la previsión de extender la indemnización por extinción contractual también a esta situación resultante de la paralización temporal. Previsión que, por cierto, entendemos ligada a la consideración de esta paralización temporal como causa de extinción.

Lo cierto es que el régimen jurídico a estudio participa en este extremo de una naturaleza híbri$\mathrm{da}$, como sucede en tantas ocasiones en nuestra disciplina (el convenio colectivo estatutario, considerando su posible contenido obligacional; los criterios a efectos de su interpretación; la naturaleza del despido disciplinario; etc.). A su naturaleza última suspensiva, y consiguiente obligación empresarial -modulada respecto de la regla general de la reserva del puesto de trabajo, algo tampoco desconocido- de una, llamémosla, reactivación laboral, se aúnan los efectos propios de una extinción contractual, dado el carácter indemnizado convencionalmente establecido. Sea uno u otro el tratamiento, el art. 267 LGSS parece incluir en todo caso como situación legal de desempleo esta situación, siquiera la referencia a la extinción del contrato de obra o servicio determinado sea más clara.

Si observamos su extinción más allá de lo anterior, desde el año 2007 se advierte expresamente en la negociación colectiva sectorial estatal que, en cuanto a las causas y efectos de la extinción de este contrato, habrá de estarse, en concreto, a lo establecido en los artículos 49 a 57 ET y a las especialidades previstas en el convenio. Llamando el VI CGSC a respetar en todo caso la cuantía establecida en el art. 49.1 c) ET, lo que parece difícil sea de otro modo pues la especialidad consistente en establecer una indemnización del 7 por 100, contemplada en la negociación colectiva, es superior a la prevista en aquel precepto legal y constituye una especialidad de esta figura. Es decir, la indemnización es muy superior a la de 12 días de salario por año de servicio de aquel precepto.

Si argumentamos de contrario y nos preguntamos qué sucedería de no existir esta especial figura, las previsiones estatutarias demandarían la realización de un contrato independiente por cada obra o servicio determinado. Contrato que de ser único y de duración ajustada a los límites legales (o mejora negocial en su caso) conllevaría la extinción con una indemnización menor. Cada obra o servicio obligaría a formalizar un nuevo contrato, que podría recaer en el mismo trabajador o no, porque la mirada también estaría puesta en el art. 15.5 ET. Y, en todo caso, parece que la indemnización o montante suma de las indemnizaciones seguiría siendo claramente inferior.

Amén de este relevante aspecto, tenemos en los dos lados de la balanza la continuidad en el contrato versus la continuidad en el empleo. Música que nos podría sonar pues, en efecto, esta última responde a uno de los postulados del triángulo dorado de la flexiseguridad, por la que personalmente no me inclino.

Considerando todo lo anterior, entiendo que la figura a estudio sí favorece la estabilidad en el empleo de forma más clara a sus alternativas. Y, en todo caso, acredita unos estándares laborales de protección mayores ante la extinción contractual. Claro, si entendemos que su alternativa es una 
contratación temporal por obra o servicio determinado, que, en principio, considerando la duración máxima del contrato y su objeto, es lo probable legalmente hablando. Más de facto, diría yo.

Cabría pensar que esa relación alternativa fuera, que no lo creemos, indefinida de entrada. La finalización de la obra conllevaría sin embargo igualmente, grosso modo, una extinción contractual con una indemnización igualmente inferior. Cejamos aquí en el despliegue de estas apresuradas conjeturas jurídicas. La cuestión merece sin duda un estudio de mayor profundidad. Así las cosas, a este nivel de reflexión, considero la previsión del contrato fijo de obra más favorable al trabajador que sus alternativas y comparto en consecuencia el carácter proclive de nuestro TS a entender que, efectivamente, favorece de hecho la estabilidad en el empleo, siquiera por el difícil $-y$ peligrosocamino de la retorsión de la naturaleza de categorías jurídicas. 